\title{
Cable Insulation Scheme to Improve Heat Transfer to Superfluid Helium in Nb-Ti Accelerator Magnets
}

\author{
Marco La China and Davide Tommasini
}

\begin{abstract}
In superconducting magnets operating at high heat loads as the ones for interaction region of particle colliders or for fast cycling synchrotrons, the limited heat transfer capability of state-of-the-art electrical insulation may constitute a heavy limitation to performance. In the LHC main magnets, $\mathrm{Nb}$-Ti epoxy-free insulation, composed of polyimide tapes, has proved to be permeable to superfluid helium, however the heat flux is rather limited.

After a review of the standard insulation scheme for $\mathrm{Nb}-\mathrm{Ti}$ and of the associated heat transfer mechanisms, we show the existence of a large margin available to improve insulation permeability. We propose a possible way to profit of such a margin in order to increase significantly the maximum heat flux drainable from an all polyimide insulated $\mathrm{Nb}$ - $\mathrm{Ti}$ coil, as it is used in modern accelerator magnets.
\end{abstract}

Index Terms-Cable insulation, epoxy resin insulation, insulation thermal factors, polyimide films, superconducting accelerator magnets.

\section{INTRODUCTION}

$\mathbf{T}$ HE main barrier to heat extraction from superconducting cables is represented by electrical insulation. Every turn of the coil is in fact dielectrically insulated from the adjacent turns as well as from the metallic components of the magnet.

The current state-of-the-art insulation scheme, used in the main LHC superconducting Nb-Ti dipoles, is composed of helicoidally wounded polyimide tapes that allow superfluid helium penetrating inside the coil.

We review the standard insulation scheme for $\mathrm{Nb}$-Ti magnets (Section II) and we present an analytical heat transfer model (Section III). We then propose a new insulation scheme (Section IV) that greatly enhances permeability to superfluid helium and we compare it to the state-of-the-art insulation (Section V). We report the first experimental results on the new insulation schemes (Section VI) and we draw conclusions (Section VII).

\section{State-OF-THE-ART ALL-Polyimide InSUlation}

The cable insulation developed for the LHC magnets [1], [2] and shown in Fig. 1, is the result of work started in the early 70's for the ISR [3], passing through SSC [4], HERA [5] and RHIC [6]. It consists of polyimide tapes arranged in layers helically wound around the cable.

The first, inner, layer has the function to dielectrically insulate adjacent turns. The inter-turn voltage that arises in case of a quench is around $100 \mathrm{~V}$ and the current can make its way across

Manuscript received August 22, 2007

The authors are with CERN, CH-1211, Geneva 23, Switzerland (e-mail: marco.la.china@cern.ch; davide.tommasini@cern.ch).

Color versions of one or more of the figures in this paper are available online at http://ieeexplore.ieee.org.

Digital Object Identifier 10.1109/TASC.2008.920574

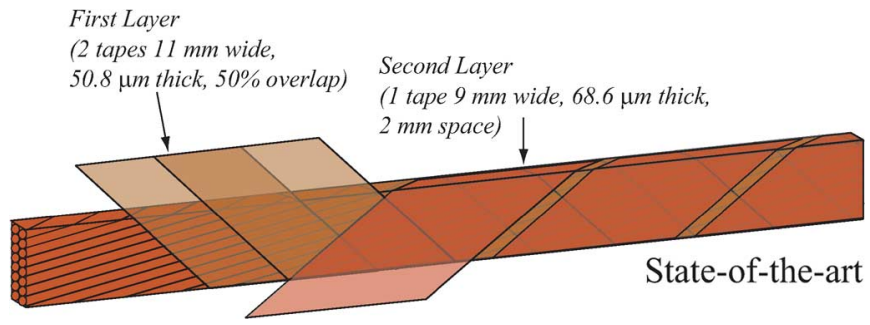

Fig. 1. State-of-the-art all-polyimide insulation developed for LHC main dipoles.
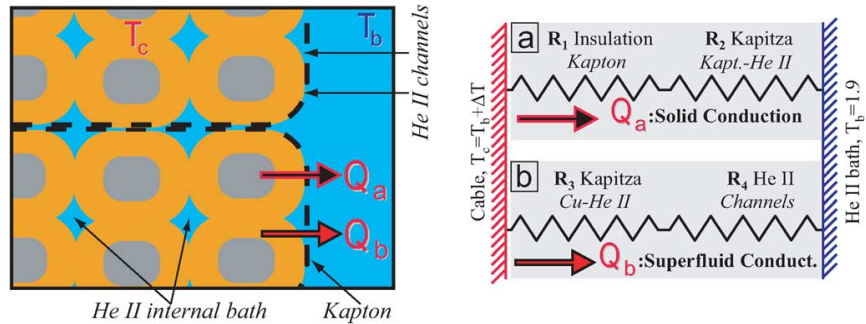

Fig. 2. Schematization of heat transfer path through porous insulation.

the insulation in case of material defects (punch-through), or along the insulation surface. To reduce the punch-through risk and to provide a sufficiently long surface path, the first layer is composed by two $50 \%$ overlapped tapes.

The second, outer, layer is intended to protect the first layer and provide cohesion between coil turns. To fulfill all these requirements, the second layer consists of a single, thicker polyimide tape with, on the outer face, a thin coat of polyimide adhesive activated by thermal treatment; the tape is wounded with spacing to create gaps for helium to reach the inner layer.

\section{Heat TRANSFER MODEL}

In a permeable insulation helium can enter and fill meanders between strands forming an internal bath (Fig. 2); such an internal bath is connected to the external bath through a network of micro-channels between insulation wraps. Heat from the strands to the external bath can thus follow two parallel paths: one formed by helium channels (superfluid conduction path for LHC); the other path being the solid conduction through insulation material. In Fig. 2 we show a representation of the two parallel paths in terms of equivalent thermal resistances.

We use such a schematization to model heat transfer across $\mathrm{Nb}$-Ti insulation under the following assumptions:

- longitudinal heat transfer plays a role only in the estimate of the equivalent channel size,

- heat is evacuated through the cable small face (Fig. 2), 


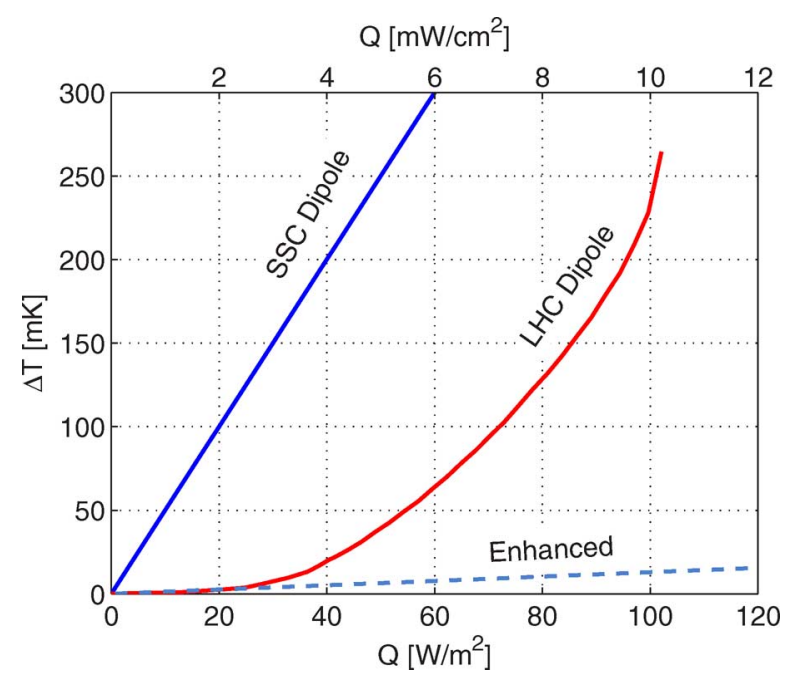

Fig. 3. Heat transfer curves for SSC and LHC insulations (measured [11]), and for enhanced insulation (estimated), all data refer to He II.

- network of channels can be treated as a single channel with equivalent cross section,

- strands, internal bath and external bath are isothermal,

- the two parallel paths are decoupled,

- thermal boundary resistance at the strand-insulation interface is negligible [7].

Thanks to the very high thermal conductivity of helium, superfluid conduction path is characterized by very low thermal impedance. However heat transport through superfluid helium channels is limited by the lambda transition (He II turns into He I) at the channel inlet [8]: the much poorer thermal conductivity of He I causes in fact a drastic reduction of heat transport through the channel that is thus considered saturated.

Thermal impedance and saturation level depend on channel geometries (cross-section, length) and bath temperature [9] but the topology of porous insulation does not allow a direct estimate or measurement of channel dimensions. However experimental evaluation of heat transfer can be found in literature [10], [11], and, even if the data cannot be directly compared between each other, being based on different experimental set-up, they confirm the remarkable efficiency of a porous insulation for small increase of cable temperature.

As an example we report data published by C. Meuris [11] during the development of the LHC main dipole insulation. Heat transfer across insulation was evaluated by measuring the temperature rise of a solid cable mock-up made of steel, heated by joule-effect, immersed in a superfluid helium bath and insulated using different insulation schemes.

Solid curves in Fig. 3 represent mock-up temperature increase versus heat flux for two types of all-polyimide insulation with two distinct levels of permeability to helium: SSC dipole type and LHC dipole type. SSC dipole insulation scheme was designed to operate in supercritical helium at $4.2 \mathrm{~K}$ disregarding permeability criteria whereas LHC dipole insulation is permeable to helium thanks to the tape spacing in the outer layer. We also report, as dashed line, the estimated heat transfer achievable with the enhanced insulation detailed in Section IV.

The very small permeability of SSC dipole type insulation is remarked by the linear temperature heat relation featured by

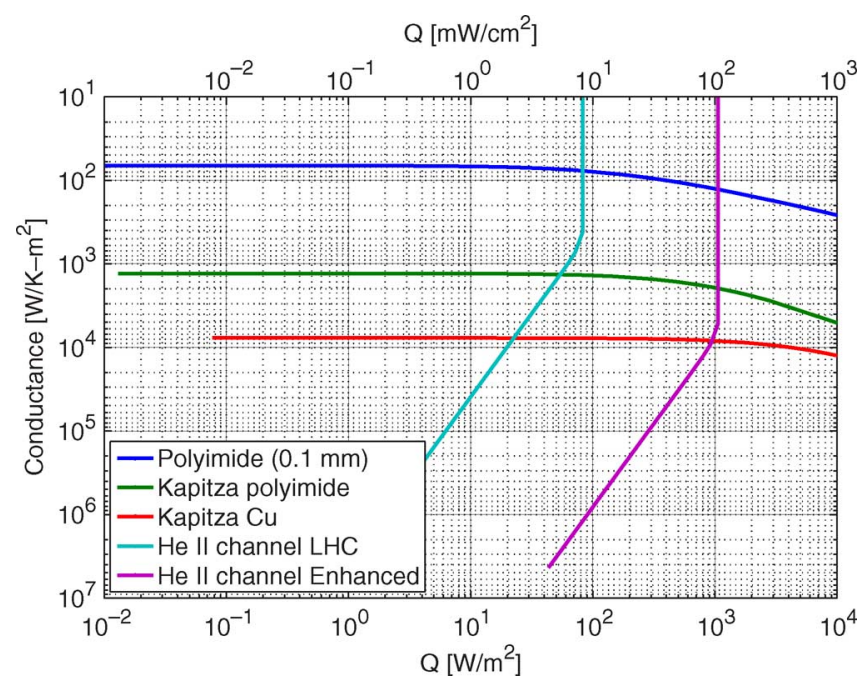

Fig. 4. Equivalent thermal resistances of insulation components in a $1.9 \mathrm{~K}$ helium bath.

the corresponding curve; its constant slope is in fact related to conduction through solid. The LHC curve, instead, is strongly non-linear: temperature rise is very small for small heat loads and grows fast for higher loads. For example a temperature increase of $10 \mathrm{mK}$ permits the evacuation of $34 \mathrm{~W} / \mathrm{m}^{2}$ for LHC versus only $2 \mathrm{~W} / \mathrm{m}^{2}$ for SSC.

To compare the weight, from a thermal point of view, of the different coil components with typical dimensions, we plot, in Fig. 4, the equivalent thermal resistances versus heat flux in a $1.9 \mathrm{~K}$ isothermal bath of helium.

Conductivity of polyimide (Kapton) has been measured by Lawrence [12], boundary resistance at the $\mathrm{Cu}-\mathrm{He}$ II interface is taken from [13] and at the polyimide-He II interface from [14].

The resistance through the bulk material is computed considering a polyimide thickness of $0.1 \mathrm{~mm}$. The resistance of helium channels is given for two different levels of permeability: corresponding to the LHC type insulation and to the enhanced insulation scheme described later (Section IV). It can be noticed that solid conduction is the most limiting factor for heat transfer in the region of interest and that helium channels offer a very small thermal resistance that grows fast with heat flux until saturation is reached.

\section{ENHANCED INSULATION DETAILS}

In state-of-the-art insulation schemes for $\mathrm{Nb}$ - Ti, the main limitation to heat transfer is due to the overlapped wrapping of the tapes in the first layer. As shown in Fig. 1 in fact, the tapes are overlapped leaving in principle no space for cooling channels. However the first layer always features a limited degree of permeability because helium, thanks to its very low viscosity, can filter between the tapes.

The second layer is wound with spacing to form channels to let helium easily reach the first insulation layer. In the LHC main magnets these channels have also the role of allowing a radial helium path from the inner layer coil to the outer layer coil. Yet, the efficiency of spacing in the second layer can be reduced or cancelled by other factors as epoxy glue or polyimide glue flow [15], [16]. 


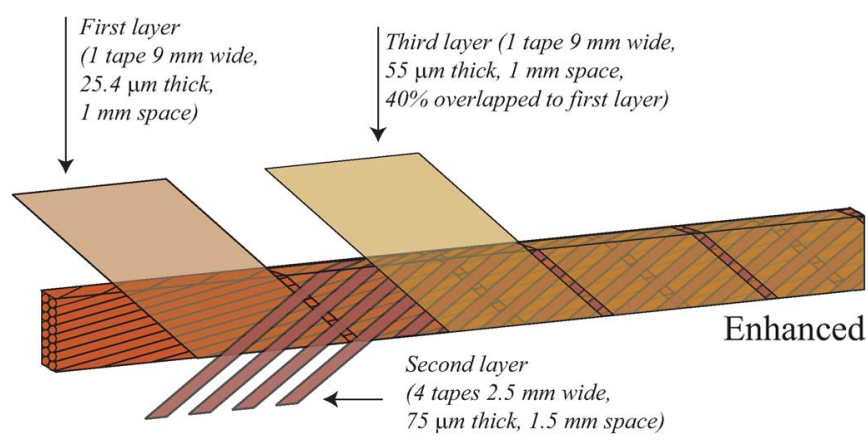

Fig. 5. Enhanced all-polyimide insulation layout. Overlap is present between first and third layer.

TABLE I

LAYER TOPOLOGIES AND FUNCTIONS IN STATE-OF-THE-ART (LHC TYPE) AND ENHANCED INSULATION SCHEMES

\begin{tabular}{cccc}
\hline \hline & $1^{\text {st }}$ layer & $2^{\text {nd }}$ layer & $3^{\text {rd }}$ layer \\
\hline \hline $\begin{array}{l}\text { State-of- } \\
\text { the-art }\end{array}$ & $\begin{array}{c}2 \text { tapes with } \\
\text { overlap }\end{array}$ & $\begin{array}{c}1 \text { tape with } \\
\text { spacing }\end{array}$ & - \\
& (DP) & (HC,MC) & \\
\hline \multirow{3}{*}{ Enhanced } & $\begin{array}{c}1 \text { tape with } \\
\text { spacing }\end{array}$ & $\begin{array}{c}4 \text { tapes with } \\
\text { spacing }\end{array}$ & $\begin{array}{c}\text { 1 tape with } \\
\text { spacing }\end{array}$ \\
& (DP, HC) & (HC) & (DP,HC,MC) \\
\hline \hline
\end{tabular}

Function legend:DP = Dielectric Protection,

MC = Mechanical protection and Cohesion,

$\mathbf{H C}=$ Helium Channels

For what concerns the compression once the coil is in operation, its effect on permeability should be remarkable only between 0 and $10 \mathrm{MPa}$ where most of positioning of polyimide between cable strands takes place. Above $10 \mathrm{MPa}$ the LHC coils show a relatively high elastic modulus, of the order of $10 \mathrm{GPa}$, which indicates that the morphology of the insulation wraps remains relatively stable.

The baseline concept to enhance insulation permeability is to create channels connecting internal and external helium baths. In the proposed scheme, the two overlapped tapes, originally belonging to the first layer, are split and separated by a series of polyimide strips wound counter-wise, as shown in Fig. 5.

In such a configuration three distinct layers, all wound with spacing, can be identified and tape overlap exists between tapes of the first and third layer ("spaced overlap"). Such solution is significantly innovative: in all insulations up to date the overlap is between tapes of the same, inner, layer. The third layer tape, thicker and coated with adhesive on the external side, provides mechanical protection, dielectric protection and cohesion between turns. Characteristics of state-of-the-art and enhanced insulation can be compared in Table I.

In the next section we will compare the expected heat transfer achievable with LHC, SSC and enhanced insulations.

\section{HeAt TRANSFER COMPARISON}

To estimate heat transfer in operating conditions it is necessary to combine the thermal properties of the insulation with the superconducting properties of the cable. The maximum $\Delta \mathrm{T}$ exploitable for heat transfer is in fact the temperature margin of

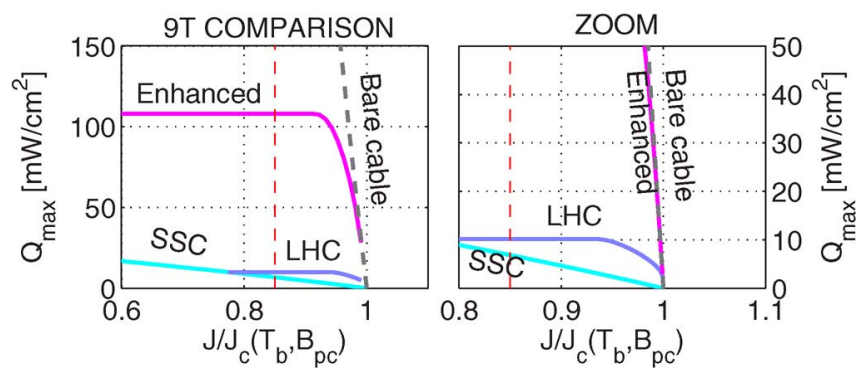

Fig. 6. Maximum heat flux versus critical current density (normalized to critical one). Cable field $9 \mathrm{~T}$, bath temperature $1.9 \mathrm{~K}$.

the cable $\left(\mathrm{T}_{\text {critical }}-\mathrm{T}_{\text {bath }}\right)$ that is function of magnetic field and of current density.

In Fig. 6 we plot, for three insulations with different levels of permeability, the maximum allowed heat flux $\mathrm{Q}_{\max }$ transferred from a $\mathrm{Nb}$-Ti coil immersed in a $1.9 \mathrm{~K}, 1$ bar helium bath, at a magnetic field of $9 \mathrm{~T}$, versus the ratio $\mathrm{J} / \mathrm{J}_{\mathrm{c}} . \mathrm{J}$ is the cable current density and $\mathrm{J}_{\mathrm{c}}$ is the critical current density at $1.9 \mathrm{~K}$ and $9 \mathrm{~T}$.

Two of the three insulations considered are the ones presented in Section III (SSC and LHC) whereas the third is the enhanced insulation presented in Section IV. We also plot the heat transfer in case of insulation with infinite permeability (bare cable); this is an asymptotic limit computed by taking into account a cylindrical conductor immersed in a $1.9 \mathrm{~K}$ helium bath.

In the non-permeable insulation (SSC) the maximum heat flux is linearly related to temperature margin. In permeable insulations, instead, the strongly non-linear $\Delta \mathrm{T}-\mathrm{Q}$ relation (shown in Fig. 3) allows a larger heat transfer at small temperature margin; this explains the steep initial slope of the LHC and Enhanced curves.

Permeability of SSC and LHC insulations has been experimentally evaluated [11] whereas permeability of the enhanced insulation has been computed. The enhanced insulation, thanks to its "spaced overlap" topology, allows estimating a priori the size of helium channels. Heat transfer was computed assuming a fully developed Gorter-Mellink [17] regime in the channels; the effect of coil compression was implemented in the model as a reduction of channel dimensions. As shown in Fig. 6 at 9 T, $85 \% \mathrm{~J}_{\mathrm{c}}$ (dot-dash vertical line) there is a factor of ten between the maximum heat transfer achievable with state-of-the-art and with enhanced insulation.

\section{FIRST EXPERIMENTAL RESUltS}

We report the first outcomes of the program of tests conceived to progressively validate all the different aspects of the enhanced insulation design.

The development of a purposely conceived porosity meter device allowed us to compare different insulation schemes applied to Rutherford cables. The porosity meter uses air at ambient temperature instead of superfluid helium: in this way the experimental set-up complexity is drastically reduced. The baseline concept is that both air mass flow and helium heat flow increase with channel cross areas.

The porosity meter is a mould designed to force a flow through a $12 \mathrm{~cm}$ long cable stack, shown in Fig. 7, along two alternative paths: longitudinal and radial. In the longitudinal 


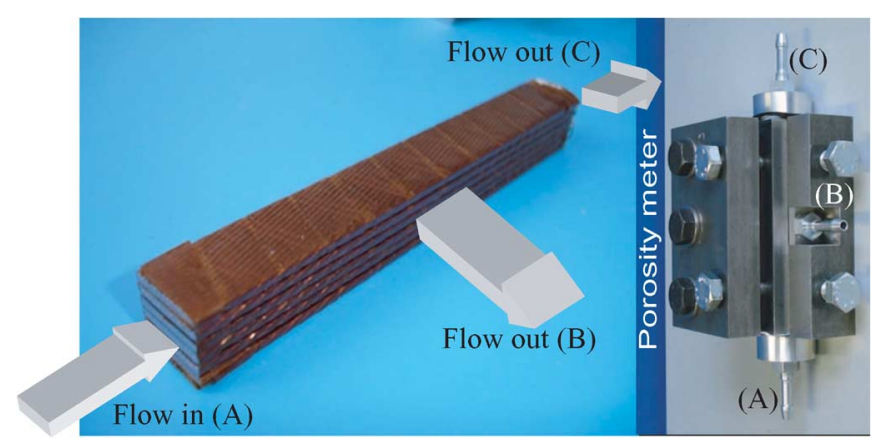

Fig. 7. Cured stack of insulated cables. Radial and longitudinal flow paths are shown.

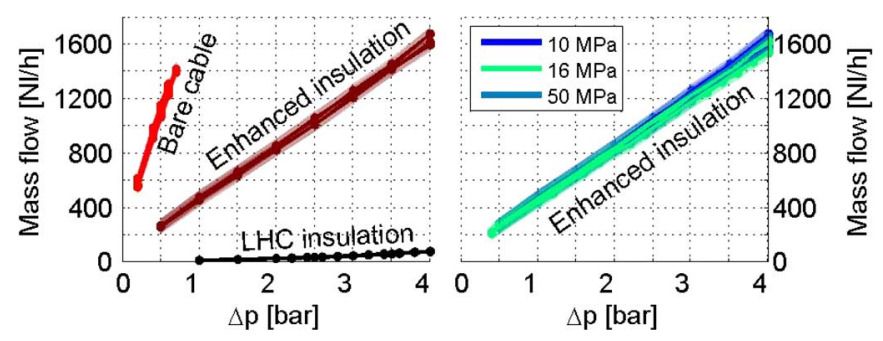

Fig. 8. Radial flow in cables with vertical compression of $10 \mathrm{MPa}$ (left plot). Radial flow in cables with enhanced insulation, vertical compression of: 10, 16 and $50 \mathrm{MPa}$ (right plot).

path, air crosses the cable stack from end to end (flow in A, flow out C, Fig. 7), without passing through the insulation.

In the radial path, instead, air enters the stack from one end and exits laterally, at half way, thus crossing the insulation (flow in A, flow out B, Fig. 7). Insulation porosity is estimated from the comparison between radial and longitudinal flows.

We tested cables of LHC Main Dipole Inner Layer type, arranged in 6 units-stacks, manufactured following the standard bonding cycle of the LHC coils, in particular maximum pressure of $80 \mathrm{MPa}$ and temperature of $190^{\circ} \mathrm{C}$. For each tested insulation we measured the air mass flow along longitudinal and radial paths induced by inlet pressures ranging from 1.2 to 5.0 bar with the outlet at atmospheric pressure (1 bar). In Fig. 8, left, we plot the measured air mass flows versus relative pressure, that is the difference between inlet and outlet pressure.

The three curves refer to the radial flow across a stack of cable with no insulation, LHC insulation and enhanced insulation; all stacks are vertically compressed at $10 \mathrm{MPa}$. The slope of each curve is a direct index of porosity to air: enhanced insulation shows a degree of porosity at least one order of magnitude bigger than LHC insulation and one smaller than the bare cable. In Fig. 8, right, we show the influence of vertical compression on the new insulation porosity. The three practically identical curves refer to radial porosity under 10, 16 and $50 \mathrm{MPa}$. It can be seen that the porosity measured at $10 \mathrm{MPa}$ is confirmed at 16 and at $50 \mathrm{MPa}$ meaning that the network of channels between insulation wraps in the "spaced overlap" configuration is still effective. In the second part of the experimental program we will verify the dielectric insulation, the resistance to small radius bends and the actual heat transfer in superfluid helium at $1.9 \mathrm{~K}, 1$ bar.

\section{CONCLUSIONS}

We presented a new all-polyimide insulation scheme designed to increase heat transfer in $\mathrm{Nb}-\mathrm{Ti}$ magnets. Such insulation features a remarkable permeability to helium thanks to the newly introduced "spaced overlap" concept. Model-based comparison with state-of-the-art insulation shows, at 9 T, 85\% $\mathrm{J}_{\mathrm{c}}$, a factor of ten improvement in heat transfer when compared to the LHC type scheme.

We tested the permeability to air and we measured a factor ten improvement with respect to state-of-the-art insulation, unchanged for vertical compressions in the 10 to $50 \mathrm{MPa}$ range. If He II heat transfer tests on coil block will confirm such a performance, and if electrical tests in actual conditions will prove its robustness, new opportunities and flexibility will be offered to designers for the implementation of new generation interaction region for the LHC and for fast cycled superconducting magnets.

\section{REFERENCES}

[1] CERN, "LHC design report," vol. 1, CERN-2004-003-V1, 2004.

[2] L. Rossi, "State-of-the art superconducting accelerator magnets," IEEE Trans. Appl. Supercond., vol. 12, pp. 219-227, Mar. 2002.

[3] J. Billan, "Selection of superconducting wires for D.C. magnets," presented at the Int. Symp. High Voltage Insulation for Low Temperature Application, Wroclaw, Sep. 13-17, 1976, Also in: CERN-ISRLTD-76-15.

[4] T. S. Jaffrey et al., "Fermilab-built SSC collider dipoles using low temperature curing insulation systems with and without glass tape," in Proc. 15th Particle Accelerator Conf. PAC'93, Washington, DC, 1993, pp. 2769-2771.

[5] C. H. Dustmann, M. Forster, and D. Bonmann, "Series production of the superconducting HERA dipole magnets," IEEE Trans. Magn., vol. 24, pp. 1338-1341, Mar 1988.

[6] M. Anerella et al., "Improved cable insulation for superconducting magnets," in Proc. 15th Particle Accelerator Conf. PAC'93, Washington, DC, 1993, pp. 2790-2792.

[7] D. S. Matsumoto, C. L. Reynolds, Jr, and A. C. Anderson, "Thermal boundary resistance at metal-epoxy interfaces," Phys. Rev. B, vol. 16, pp. 3303-3307, Oct. 1977.

[8] V. Arp, "Heat transport through helium II," Cryogenics, vol. 10, pp. 96-105, Apr. 1970.

[9] S. W. Sciver, Helium Cryogenics. New York: Plenum, 1986, pp. $142-146$.

[10] N. Kimura et al., "Heat transfer from insulated Rutherford cables immersed in pressurized He II," presented at the Cryogenic Engineering Conference, Portland, OR, Jul. 28-Aug. 1 1997, Paper ACR-J-19.

[11] C. Meuris, B. Baudouy, D. Leroy, and B. Slezess, "Heat transfer in electrical insulation of LHC cables cooled with superfluid helium," Cryogenics, vol. 39, pp. 921-931, Nov. 1999.

[12] J. Lawrence, A. B. Paterl, and J. G. Brisson, "The thermal conductivity of Kapton HN between 0.5 and 5 K," Cryogenics, vol. 40, pp. 203-207, Mar. 2000

[13] A. Kashani and S. W. Van Sciver, "High heat flux Kapitza conductance of technical copper with several different surface preparations," Cryogenics, vol. 25, pp. 238-242, May 1985.

[14] B. Baudouy, "Kapitza resistance and thermal conductivity of Kapton in superfluid helium," Cryogenics, vol. 43, pp. 667-672, Dec. 2003

[15] B. Baudouy, M. X. François, F.-P. Juster, and C. Meuris, "He II heat transfer through superconducting cables electrical insulation," Cryogenics, vol. 40, pp. 127-136, Feb. 2000.

[16] L. Chiesa et al., "Thermal studies of a high gradient quadrupole magnet cooled with pressurized, stagnant superfluid," IEEE Trans. Appl. Supercond., vol. 11, pp. 1625-1628, Mar. 2001.

[17] J. Gorter and J. H. Mellink, "On the irreversible processes in liquid helium II,” Physica, vol. 15, pp. 285-304, May 1949. 